\title{
Analysis on Existing Basic Slas and Green Slas to Define New Sustainable Green SLA
}

\author{
Iqbal Ahmed \\ Graduate School of Science and \\ Engineering, Saga University, Japan
}

\author{
Hiroshi Okumura \\ Graduate School of Science and \\ Engineering, Saga University, Japan
}

\author{
Kohei Arai \\ Graduate School of Science and \\ Engineering, Saga University, Japan
}

\begin{abstract}
Nowadays, most of the IT (Information Technology) and ICT (Information and Communication Technology) industries are practicing sustainability under green computing hoods. Users/Customers are also moving towards a new sustainable society. Therefore, while getting or providing different services from different ICT vendors, Service Level Agreement (SLA) becomes very important for both the service providers/vendors and users/customers. There are many ways to inform users/customers about various services with its inherent execution functionalities and even non-functional/Quality of Service (QoS) aspects through SLAs. However, these basic SLAs actually do not cover eco-efficient green issues or ethical issues for actual sustainable development. That is why green SLA (GSLA) should come into play. GSLA is a formal agreement incorporating all the traditional/basic commitments as well as respecting the ecological, economical and ethical aspects of sustainability. This research would survey on different basic SLA parameters for various services in ICT industries. At the same time, this survey would focus on finding the gaps and incorporating basic SLA parameters with existing green computing issues and ethical issues for different services in various computing domains. This research defines future GSLA in relationship with ICT product life and three pillars of sustainability. The proposed definition and overall survey could help different service providers/vendors to define their future GSLA as well as business strategies for this new transitional sustainable society.
\end{abstract}

Keywords-SLA; GSLA; Green ICT; Sustainability; IT ethics; ICT Product Life

\section{INTRODUCTION}

SLA is defined as a formal document between an IT service provider and one or more customer outlining Service Commitment [1]. The main issue is that most of these traditional/basic SLA actually do not cover eco-efficient green issues. Currently, cloud and grid computing and many data centers acts as most promising service providers. These computing and communication industry provides different services in compare to traditional computing with some scalability benefits. At the same time, cloud services are offered at various levels: Infrastructure, Platform and Software as a Service [2]. At each level, they maintain a SLA with respect to their parties. Therefore, this shows the growth rate of SLA in recent time as well as the need of GSLA for actual sustainability achievement in the industry. Presently, the revolution of ICTs and ITs in daily average life has also resulted in the increase of Green House Gas (GHG), due to continual increase in global "carbon footprint"'. In 2007, the ICT sector produced as much GHG as the aero industry and is projected to grow rapidly $[3,4]$. If ICT has a negative impact on environment, it can be also be used for greening the other human activities (logistic, city, industry etc) in this new society. Indeed, the dimensions of Green Informatics contributions are: the reduction of energy consumption, the rise of environmental awareness, the effective communication for environmental issues and the environmental monitoring and surveillance systems, as a means to protect and restore natural ecosystems potential [5]. At the same time, many IT and ICT industries or service providers need to think about their business scope in the light of green perspective. However, the IT and ICT sectors mostly concern about energy or power consumption, carbon, recycling and productivity issues under greening computing lens. On the contrary, most of the recent industries overlooked many green parameters under sustainability lens. Therefore, with the increase attention that green informatics and sustainability practice within our society, it is timely to not only conduct SLAs for traditional/basic computing performance metrics or only on energy or carbon footprint issues, but also to relate the effort of conducting green computing with respect to $3 \mathrm{Es}$ of (Ecology, Economy and Ethics) sustainability pillars. Therefore, the journey of GSLA is getting importance in ICT business world. This research did thorough review on existing basic SLA indicators for network, storage, compute and multimedia domain in IT industry. Then, it goes deep down for finding more current green performance indicators in some datacenter's SLAs. In addition, a new future GSLA definition proposed, which shows the importance and relationships of ICT product life cycle. Moreover, the GSLA should be designed considering three pillars of sustainability. Finally, GSLA research briefly describes the management complexities and some challenges.

The rest of the work is organized according to 4 sectionsthe next Research Review section discusses and analyses some existing scientific theory and practical works based on basic SLA for four different services in the industry. Empirical Work Review section indentifies all basic SLA indicators for network, compute, storage and multimedia services, which do not cover any eco-efficient parameters. Next, the following subsection discovers most of the green indicators for various services, usually used in grid and cloud computing, datacenters etc. Basic SLA and existing GSLA parameters are also derive and organize in details through existing empirical viewpoint. The existing GSLA subsection actually shows currents trends of the industry to practice sustainability under greening lens. The future GSLA definition sections describe the gap between greening and sustainability in the current 
industry. In addition, this section gives some hints about future indicators for sustainable future GSLA. Moreover, it also depicts the relationships of future GSLA with ICT product life in the industry. Finally, the conclusion gives brief discussion about few challenges for the ICT engineer to incorporate and trade-off between all existing indicators and new indicators for sustainable achievement in the ICT industry.

\section{RESEARCH REVIEW}

This GSLA work did rigorous literature review and analysis based on existing work in the field of SLA, GSLA, green computing, energy optimization in IT industry, impact of ICT on environment and natural resource, IT ethics issues, IT for Sustainability etc. In the findings, GSLA research divides its work based on basic SLA and then existing GSLA for various types of services from their providers. The existing theory work on basic SLA and GSLA discusses in the following sub sections.

\section{A. Basic SLAs}

S. A. Baset [6] gave an idea for presenting SLA for different cloud service providers. He surveyed on some well known public IaaS providers and found a common anatomy of basic SLA with some common metrics. In [7], H. Lee et al. offered a general SLA monitoring system architecture that could be used to monitor service levels provided by some network, Internet and application service providers. Their work showed much clear idea of finding some QoS parameters, measurement metrics for various services. In contrast, L. Jin et al. [8] presented another approach to model and understand the relationship between customers and some web service providers, which is very important for designing basic SLA and Green SLA. A. Paschke et al. contributed to a systematic categorization of basic SLA contents with a particular focus on SLA metrics in IT industry [9]. They categorized five basic IT object classes and their performance indicators in SLA. J. Lankinen et al. [10] surveyed on security profiles of some existing well known storage service providers like Amazon, Apple iCloud, Dropbox etc. In [11], the paper presented SLA for voice and Internet services covering basic performance indicators. Most of the paper found on basic SLA discussed performance based indicators for various services in recent ICT arena. Some empirical work found on SLA implementation, management, automation, template design and assessment in the context of business requirement. Very few scientific works found on interesting aspects such as security and privacy issues on traditional SLA, which could be important for green SLA research under IT ethics concept. Table I shows the brief idea of basic SLA work through some interesting criteria of SLAs as column subheads. The cell identified with " $\mathrm{X}$ " symbol means that, the authors mentioned and worked on that criteria of basic SLAs.

TABLE I. ANALYSIS OF EXISTING BASIC SLA WORKS

\begin{tabular}{|c|c|c|c|c|c|c|c|}
\hline \multirow{2}{*}{$\begin{array}{l}\text { Author } \\
\text { Lists }\end{array}$} & \multicolumn{7}{|c|}{ Analysis Criteria } \\
\hline & Services & Information & Methodology & Implementation & Assessment & Monitoring & Reuse to Green SLA \\
\hline S. A. Baset [6] & $\mathrm{X}$ & & $\mathrm{X}$ & $\mathrm{X}$ & & $\mathrm{X}$ & \\
\hline H. Lee et al. [7] & $\mathrm{X}$ & $\mathrm{X}$ & & $\mathrm{X}$ & $\mathrm{X}$ & $\mathrm{X}$ & \\
\hline L. Jin et al. [8] & $\mathrm{X}$ & $\mathrm{X}$ & $\mathrm{X}$ & $\mathrm{X}$ & & & \\
\hline A. Paschke et al. [9] & & $\mathrm{X}$ & $\mathrm{X}$ & & $\mathrm{X}$ & & \\
\hline J. Lankinen et al. [10] & $\mathrm{X}$ & $\mathrm{X}$ & & & $\mathrm{X}$ & & $\mathrm{X}$ \\
\hline Anonymous [11] & $\mathrm{X}$ & $\mathrm{X}$ & & & & & $\mathrm{X}$ \\
\hline C. Raibulet et al. [12] & & & & $\mathrm{X}$ & $\mathrm{X}$ & & \\
\hline V. Stantchev et al. [13] & & $\mathrm{X}$ & $\mathrm{X}$ & & & & $\mathrm{X}$ \\
\hline N.J. Dingle et al. [14] & & $\mathrm{X}$ & & & & $\mathrm{X}$ & $\mathrm{X}$ \\
\hline T. Unger et al. $[15]$ & & $\mathrm{X}$ & $\mathrm{X}$ & & & & \\
\hline E. Marilly et al. [16] & $\mathrm{X}$ & $\mathrm{X}$ & & $\mathrm{X}$ & & $\mathrm{X}$ & \\
\hline T. Onali [17] & $\mathrm{X}$ & $\mathrm{X}$ & & $\mathrm{X}$ & & & \\
\hline H. Ludwig et al. [18] & $\mathrm{X}$ & & & $\mathrm{X}$ & & & $\mathrm{X}$ \\
\hline P. Hasselmeyer et al. [19] & $\mathrm{X}$ & & & $\mathrm{X}$ & $\mathrm{X}$ & $\mathrm{X}$ & \\
\hline Anonymous [20] & & $\mathrm{X}$ & $\mathrm{X}$ & & & & \\
\hline E. Wustenhoff [21] & & & & & & $\mathrm{X}$ & \\
\hline Anonymous [22] & & $\mathrm{X}$ & & & & & $\mathrm{X}$ \\
\hline
\end{tabular}

B. Green SLAs

S. Klingert et al. [23] introduced the notion of Green SLAs. However, their work focused on indentifying known hardware and software techniques for reducing energy consumption and integrating green energy. In [4] and [5], the authors showed the impact of ICT in a natural environment and resources in this world. Z. S. Andreopoulou [5] proposed a model ICT for Green and Sustainability whereas SMART 2020 report [4] gave the idea of GHG emission from the ICT sector. G. V. Laszewski et al. [24] invented a framework towards the inclusion of Green IT metrics for grids and cloud computing. According to Md. E. Haque et al. [25], high performance computing cloud providers offer a new class of green services in response to practicing explicit sustainability goals in their field. R. R. Harmon et al. [26] defined the term Green Computing as the practice of maximizing the efficient use of computing resources to minimize environmental impact. They also discovered that, sustainable IT services require the integration of green computing practice such as power management, virtualization, cooling technology, recycling, electronic waste disposal and optimization of IT infrastructure. Finally, the white paper [22] provided some qualitative parameters in cloud service SLA which was very important for proposing Green SLA. In [27] and [28], the authors discussed one of the most promising concepts in Green SLA- IT Ethics issues. In their research, they showed the concepts of organizing ethics programs in IT industry. The 
existing scientific work on green SLA is mainly based on cloud and grid computing environment. Some works have been found on green services, operation and framework for the cloud infrastructure [30]; few work done on green performance indicators for designing SLA. The next Table II demonstrates the analysis of exiting green SLA works with some criteria, such as green services and operations, greening practice, green metrics, framework development and monitoring. Here some papers also discussed IT ethics issues briefly. Therefore, IT ethics need to include here as an important analyzing criteria in the table.

TABLE II. ANALYSIS OF EXISTING GSLA WORKS

\begin{tabular}{|c|c|c|c|c|c|c|}
\hline \multirow[b]{2}{*}{$\begin{array}{l}\text { Author } \\
\text { Lists }\end{array}$} & \multicolumn{6}{|l|}{ Analysis Criteria } \\
\hline & $\begin{array}{l}\text { Green } \\
\text { Services \& Operations }\end{array}$ & $\begin{array}{l}\text { Greening } \\
\text { Computing Practice }\end{array}$ & $\begin{array}{l}\text { Metrics } \\
\text { Information }\end{array}$ & $\begin{array}{l}\text { Framework/ } \\
\text { Implementation }\end{array}$ & Assessment & $\begin{array}{l}\text { IT Ethics } \\
\text { issue }\end{array}$ \\
\hline L. Wu et al. [4] & & $\mathrm{X}$ & & & & $\mathrm{X}$ \\
\hline Z. S. Andreopoulou [5] & & $\mathrm{X}$ & & & & $\mathrm{X}$ \\
\hline Klingert et al. [23] & $\mathrm{X}$ & $\mathrm{X}$ & & & & \\
\hline G. V. Laszewski et al. [24] & $\mathrm{X}$ & $\mathrm{X}$ & $\mathrm{X}$ & $\mathrm{X}$ & & \\
\hline Md. E. Haque et al. [25] & & & & $\mathrm{X}$ & $\mathrm{X}$ & \\
\hline R. R. Harmon et al. [26] & $\mathrm{X}$ & $\mathrm{X}$ & $\mathrm{X}$ & & & \\
\hline Fritz H. Grupe et al. [27] & & & & $\mathrm{X}$ & & $\mathrm{X}$ \\
\hline R. Herold [28] & & & & & & $\mathrm{X}$ \\
\hline N. Agarwal et al. [29] & $\mathrm{X}$ & $\mathrm{X}$ & & & & \\
\hline Ahmed et al. [30] & $\mathrm{X}$ & $\mathrm{X}$ & & $\mathrm{X}$ & $\mathrm{X}$ & \\
\hline Li et al. [31] & & $\mathrm{X}$ & & $\mathrm{X}$ & & \\
\hline Kien Le et al. [32] & & & & $\mathrm{X}$ & & \\
\hline M. Nichollas [33] & & & & & & $\mathrm{X}$ \\
\hline A. P Bianzino et al. [34] & $\mathrm{X}$ & $\mathrm{X}$ & & $\mathrm{X}$ & & \\
\hline A. Atrey et al. [35] & & $\mathrm{X}$ & $\mathrm{X}$ & $\mathrm{X}$ & & \\
\hline A. Orgerie [36] & $\mathrm{X}$ & $\mathrm{X}$ & & & & \\
\hline
\end{tabular}

\section{EMPIRICAL WORK REVIEW}

In the findings on existing empirical work, green SLA research splits its work based on basic SLA and then existing green SLA for various types of services from their providers such as Network, Compute, Storage and Multimedia [37].

In the basic SLA section, findings are divided into four main services as network, compute, storage and multimedia [37]. Most of the performance indicators in basic SLA sections were quantitative parameters and they were simple to evaluate, control and monitor.

\section{A. Basic SLAs for Network, Compute, Storage and Multimedia domain:}

Usually network services domain include connectivity and switching as well as advanced network systems and management functions for well known network service providers.

The basic SLA for network specifies service level commitments which are applied to measure and evaluate network performance and give proper support for their clients. Usually, from different network service provider, the following performance indicators [7, 9, 11, 24] found in their SLAs are- Network Availability, Delay, Latency, Packet Delivery Ratio, Jitter, Congestion, Flow Completion time, Response time, Bandwidth, Utilization, MTBF (Mean Time Between Failure), MTRS (Mean Time to Restore Services), Solution time, Resolution time, LAN/WAN period of operation, LAN/WAN Service Time, Internet access across Firewall, RAS (Remote access Services) (Table III).
TABLE III. BASIC SLAS FOR NETWORK SERVICES

\begin{tabular}{|l|l|l|l|}
\hline Sl.No. & Performance Indicator Name & Unit \\
\hline \multirow{2}{*}{1.} & $\begin{array}{l}\text { Network } \\
\text { Availability }\end{array}$ & $\begin{array}{l}\text { Connectivity } \\
\text { (IPPM) }\end{array}$ & (Percentage) \\
\cline { 3 - 3 } & Functionality & \\
\hline \multirow{2}{*}{2.} & Delay & $\begin{array}{l}\text { One way delay } \\
\text { RTT delay } \\
\text { (Round Trip Time) }\end{array}$ & $\begin{array}{l}\text { Time in } \\
\text { Milliseconds }\end{array}$ \\
\hline 3. & Latency & $\begin{array}{l}\text { Time in } \\
\text { Milliseconds }\end{array}$ \\
\hline 4. & $\begin{array}{l}\text { Packet Delivery Ratio(PDR) or } \\
\text { Packet Loss Ratio(PLR) }\end{array}$ & (Percentage) \\
\hline 5. & Jitter & $\begin{array}{l}\text { Time in } \\
\text { Milliseconds }\end{array}$ \\
\hline 6. & Congestion & $\%$ (Percentage) \\
\hline 7. & Flow Completion Time (FCT) & $\begin{array}{l}\text { Time in } \\
\text { Milliseconds/ } \\
\text { Seconds }\end{array}$ \\
\hline 8. & Response Time & $\begin{array}{l}\text { Time in } \\
\text { Milliseconds }\end{array}$ \\
\hline 9. & Bandwidth & Hertz (Hz) \\
\hline 10. & Utilization & (Percentage) \\
\hline 11. & LAN/WAN period of Operation & $\begin{array}{l}\text { Time in } \\
\text { Milliseconds/ Seconds }\end{array}$ \\
\hline 12. & LAN/WAN Service Time & $\begin{array}{l}\text { Time in } \\
\text { Milliseconds }\end{array}$ \\
\hline 13. & MTBF (Mean Time between Failure) & $\begin{array}{l}\text { Time in } \\
\text { Milliseconds }\end{array}$ \\
\hline 14. & MTRS (Mean Time to Restore Services) & $\begin{array}{l}\text { Time in } \\
\text { Milliseconds }\end{array}$ \\
\hline Sl.No. & Performance Indicator Name & Unit \\
\hline 15. & Solution Times & $\begin{array}{l}\text { Time in } \\
\text { Seconds/Minutes/ Hours }\end{array}$ \\
\hline 16. & Internet access across Firewall & YES/NO \\
\hline 17. & RAS (Remote Access Service) & YES/NO \\
\hline 18. & Resolution Time (TTR) & Time \\
\hline
\end{tabular}


Among these performance indicators, only Internet access across Firewall and $R A S$ are subjective indicators- there is no standard procedure to evaluate or calculate these indicators. Some indicators like Bandwidth, Utilization, and Congestion are related to link capacity whereas Availability, Delay, Jitter, Response Time etc. associated with time related information for different network service providers.

Most the cloud, grid service companies provides computing service to their consumers. In recent time, the Service Oriented Architecture (SOA) also comes into the computing field. The main point is that there is research on building middleware SLA infrastructure for computing services. Some of the current work: the European Unionfunded Framework 7 research project, SLA@SOI, which is research on aspects of multi-level, multi-provider SLAs within service-oriented infrastructure and cloud computing [38]. The basic SLA parameter [9, 11, 22, 24] for computing domains are,-: Broad Network Accessibility, Multi-tenancy, Rapid Elasticity, Scalability, Resource Pooling Time, Solution Time, Response Time, Availability (MTBF \& MTTR), Capacity, Virtualization, Delay, Resolution Time and Logging \& Monitoring. Here, Broad Network Accessibility, Multi-tenancy and Logging \& Monitoring are informative indicators presented in their SLAs (Table IV).

TABLE IV. BASIC SLAS FOR COMPUTE SERVICES

\begin{tabular}{|l|l|l|}
\hline Sl.No. & Performance Indicator Name & Unit \\
\hline 1. & Broad Network Accessibility & $\begin{array}{l}\% \text { (Percentage) } \\
\text { Or YES/NO }\end{array}$ \\
\hline 2. & Multi-tenancy & YES/NO \\
\hline 3. & Rapid Elasticity & $\%$ (Percentage) \\
\hline 4. & Scalability & (Percentage) \\
\hline 5. & Resource Pooling Time & $\begin{array}{l}\text { Time in Milliseconds } \\
\text { Or Seconds }\end{array}$ \\
\hline 6. & Solution Time & $\begin{array}{l}\text { Time in } \\
\text { Seconds/Minutes/ Hours }\end{array}$ \\
\hline 7. & Response Time & $\begin{array}{l}\text { Time in } \\
\text { Milliseconds Or } \\
\text { Microseconds }\end{array}$ \\
\hline 8. & Availability & $\begin{array}{l}\text { Time in Milliseconds Or } \\
\text { Seconds }\end{array}$ \\
\cline { 2 - 3 } & MTTR & $\begin{array}{l}\text { Number Or } \\
\text { Request per Minutes }\end{array}$ \\
\hline 9. & Capacity & \% (Percentage) \\
\hline 10. & Virtualization & Time in Milliseconds \\
\hline 11. & Delay & Time \\
\hline 12. & Service Time & YES/NO \\
\hline 13. & Logging \& Monitoring & Time \\
\hline 14. & Resolution Time (TTR) &
\end{tabular}

The storage domains are typically handled by cloud storage provider. Interestingly, today's cloud storage SLAs just ensure uptime guarantee but not data availability and data protection. In some case, traditional SLAs just mention about data storage security and backup but there is no proper authority or standard to check their commitments. Some common basic SLA performance indicator [7, 9, 11] for storage services are as follows-: Availability, Response Time, Maximum Down Time, Uptime, Failure Frequency, Period of Operation, Service Time, Accessibility, Backup, Physical Storage Backup, Transportation for Backup, Size, Data Accessibility, Security. Among all these parameters, some of them are just informative such as Accessibility, Backup,
Physical Storage Backup, Transportation for Backup, and Security (Table V). These parameters might vary according to human perspective.

TABLE V. BASIC Slas FOR STORAGE SERVICES

\begin{tabular}{|l|l|l|}
\hline Sl.No. & Performance Indicator Name & Unit \\
\hline 1. & Availability & $\%$ (Percentage) per time \\
\hline 2. & Response time & Time in Milliseconds \\
\hline 3. & Maximum down time & Time Or \% (Percentage) \\
\hline 4. & Failure Frequency & $\%$ (Percentage) \\
\hline 5. & Periods of Operation & $\begin{array}{l}\text { Time in Milliseconds/ } \\
\text { Seconds }\end{array}$ \\
\hline 6. & Service Time & Time in Hours/Day \\
\hline 7. & Accessibility & YES/NO \\
\hline 8. & Back up & YES/NO \\
\hline 9. & Physical Storage Back up & YES/NO \\
\hline 10. & Transportation of Back up & YES/NO \\
\hline 11. & Size & Number in Bytes \\
\hline 12. & Data accessibility & Number per seconds \\
\hline 13. & Security & YES/NO \\
\hline
\end{tabular}

Multimedia service domain SLAs are classified into three broad application areas- Audio, Video and Data. It is challenging to monitor and evaluate some qualitative indicator such as Mean Opinion Score (MOS) and Lip Synchronization for one way video, conferencing or in videophone. These could vary among different consumers at the same time. Most of the SLA indicators for multimedia domain for different applications are Information Loss (PLR), Jitter, One way Delay, MOS, Lip Synchronization, and Security Policy [17]. Next Table VI shows all performance indicators for multimedia services in their SLAs.

TABLE VI. BASIC SLAS FOR MULTIMEDIA SERVICES

\begin{tabular}{|c|c|c|c|}
\hline Media & $\begin{array}{l}\text { Application } \\
\text { Name }\end{array}$ & $\begin{array}{l}\text { Performance } \\
\text { Indicator Name }\end{array}$ & Unit \\
\hline \multirow{6}{*}{ Audio } & \multirow{3}{*}{$\begin{array}{l}\text { Conversational } \\
\text { Voice }\end{array}$} & $\begin{array}{l}\text { Information Loss } \\
\text { (Packet Loss Ratio) }\end{array}$ & $\begin{array}{l}\% \\
\text { (Percentage) }\end{array}$ \\
\hline & & One way Delay & $\begin{array}{l}\text { Time in } \\
\text { Milliseconds }\end{array}$ \\
\hline & & $\begin{array}{l}\text { Delay Variation } \\
\text { (Jitter) }\end{array}$ & $\begin{array}{l}\text { Time in } \\
\text { Milliseconds }\end{array}$ \\
\hline & \multirow[b]{3}{*}{ Voice Messaging } & $\begin{array}{l}\text { Information Loss } \\
\text { (Packet Loss Ratio) }\end{array}$ & $\begin{array}{l}\% \\
\text { (Percentage) }\end{array}$ \\
\hline & & One way Delay & $\begin{array}{l}\text { Time in } \\
\text { Milliseconds }\end{array}$ \\
\hline & & $\begin{array}{l}\text { Delay Variation } \\
\text { (Jitter) }\end{array}$ & $\begin{array}{l}\text { Time in } \\
\text { Milliseconds }\end{array}$ \\
\hline \multirow[b]{3}{*}{ Video } & \multirow[b]{3}{*}{ One way Video } & $\begin{array}{l}\text { Information Loss } \\
\text { (Packet Loss Ratio) }\end{array}$ & $\begin{array}{l}\% \\
\text { (Percentage) }\end{array}$ \\
\hline & & One way Delay & $\begin{array}{l}\text { Time in } \\
\text { Milliseconds }\end{array}$ \\
\hline & & $\begin{array}{l}\text { Mean Opinion } \\
\text { Score (MOS) }\end{array}$ & $\begin{array}{l}\text { Number } \\
(0 \text { to } 5)\end{array}$ \\
\hline Media & $\begin{array}{l}\text { Application } \\
\text { Name }\end{array}$ & $\begin{array}{l}\text { Performance } \\
\text { Indicator Name }\end{array}$ & Unit \\
\hline \multirow{4}{*}{ Video } & \multirow{4}{*}{ Videophone } & $\begin{array}{l}\text { Information Loss } \\
\text { (Packet Loss Ratio) }\end{array}$ & $\begin{array}{l}\% \\
\text { (Percentage) }\end{array}$ \\
\hline & & One way Delay & $\begin{array}{l}\text { Time in } \\
\text { Milliseconds }\end{array}$ \\
\hline & & $\begin{array}{l}\text { Mean Opinion } \\
\text { Score (MOS) }\end{array}$ & $\begin{array}{l}\text { Number } \\
(0 \text { to } 5)\end{array}$ \\
\hline & & Lip Synchronization & Time in \\
\hline
\end{tabular}




\begin{tabular}{|c|c|c|c|}
\hline & & & Milliseconds \\
\hline \multirow{11}{*}{ Data } & \multirow[b]{2}{*}{ Still Images } & One way Delay & $\begin{array}{l}\text { Preferred or } \\
\text { Acceptable }\end{array}$ \\
\hline & & $\begin{array}{l}\text { Information Loss } \\
\text { (Packet Loss Ratio) }\end{array}$ & $\begin{array}{l}\% \\
\text { (Percentage) }\end{array}$ \\
\hline & \multirow[b]{2}{*}{ Interactive Game } & $\begin{array}{l}\text { Information Loss } \\
\text { (Packet Loss Ratio) }\end{array}$ & $\begin{array}{l}\% \\
\text { (Percentage) }\end{array}$ \\
\hline & & One way Delay & $\begin{array}{l}\text { Time in } \\
\text { Milliseconds }\end{array}$ \\
\hline & \multirow[b]{2}{*}{ E-mail } & $\begin{array}{l}\text { Information Loss } \\
\text { (Packet Loss Ratio) }\end{array}$ & $\begin{array}{l}\% \\
\text { (Percentage) }\end{array}$ \\
\hline & & One way Delay & $\begin{array}{l}\text { Time in } \\
\text { Milliseconds }\end{array}$ \\
\hline & \multirow[b]{2}{*}{ Web-browsing } & One way Delay & $\begin{array}{l}\text { Preferred or } \\
\text { Acceptable }\end{array}$ \\
\hline & & $\begin{array}{l}\text { Information Loss } \\
\text { (Packet Loss Ratio) }\end{array}$ & $\begin{array}{l}\% \\
\text { (Percentage) }\end{array}$ \\
\hline & \multirow{3}{*}{$\begin{array}{l}\text { Transaction } \\
\text { Services } \\
\text { e.g. e-commerce, } \\
\text { ATM }\end{array}$} & $\begin{array}{l}\text { Information Loss } \\
\text { (Packet Loss Ratio) }\end{array}$ & $\begin{array}{l}\% \\
\text { (Percentage) }\end{array}$ \\
\hline & & One way Delay & $\begin{array}{l}\text { Time in } \\
\text { Milliseconds }\end{array}$ \\
\hline & & Security Policy & YES/NO \\
\hline
\end{tabular}

\section{B. Existing Green SLA (GSLA)}

Most of the GSLA performance indicator corresponds to traditional high performance distributed computing environment such as grid and cloud computing industry. Currently, several IT and ICT industries provide their GSLAs with green computing practice. GSLA survey shows that most of existing GSLAs are mainly focused on energy/ power, carbon footprint, green energy, recycling issues. Additionally, several existing GSLA also demonstrates their productivity issues with necessary monitoring unit. In recent days, various research draws attention only on minimizing energy consumption while improving networking performance on wireless connection under green computing hood [39, 40].

Table VII depicts the performance indicators and their unit for different services considering green computing practices. The table has several headings. Green Computing Domain mentions the category of green computing practices in IT industry; Performance Indicator Name is the notion which used an evaluating, monitoring metric for defining performance in GSLAs, and then their measurable unit as Unit column. All these performance indicators help various service providers and consumers either to design or to choose services mainly with respect to energy consumption, renewable energy usages, carbon emission issues and productivity issues in recent time. However, the IT industry needs to find out new services for achieving sustainability as current trends of the society shows that people are much more concerned about new issues, such as recycling, obsolescence, ICT pollution, ethical aspects etc. It is also important to mention that, monitoring of GSLA is vital to respect the services by concerned parties.
TABLE VII. PERFORMANCE INDICATOR FOR DIFFERENT SERVICES CONSIDERING EXISTING GSLA

\begin{tabular}{|c|c|c|}
\hline $\begin{array}{l}\text { Green } \\
\text { Computing } \\
\text { Domain }\end{array}$ & Performance Indicator Name & Unit \\
\hline \multirow{15}{*}{$\begin{array}{l}\text { Energy/ } \\
\text { Power }\end{array}$} & Total Power Consumption $[26,41]$ & $\begin{array}{l}\text { kW-h } \\
\text { (Kilowatt-hour) }\end{array}$ \\
\hline & $\begin{array}{l}\text { PUE (Power Usages Effectiveness ) [24, } \\
35,37,42]\end{array}$ & $\begin{array}{l}\text { Number } \\
(1.0 \text { to } \infty) \text { Or } \\
\text { Dimensionless } \\
\end{array}$ \\
\hline & $\begin{array}{l}\text { DCiE ( Data Center Infrastructure } \\
\text { Efficiency ) }[24,38,42]\end{array}$ & $\%$ (Percentage) \\
\hline & CPE (Compute Power Efficiency) [35] & Watts \\
\hline & SPECPower $[24,35]$ & Watt \\
\hline & JouleSort [26] & $\mathrm{kW} / \mathrm{J}$ \\
\hline & WUE (Water Usages Effectiveness) [35] & Liter/kW-h \\
\hline & TDP (Thermal Design Power) [42] & Watts \\
\hline & ERF (Energy Reuse Factor) [35] & $\begin{array}{l}\text { Number } \\
{[0 \text { to } 1.0]}\end{array}$ \\
\hline & ERE (Energy Reuse Effectiveness) [35] & $\begin{array}{l}\text { Number } \\
{[0 \text { to } \infty]}\end{array}$ \\
\hline & GEC (Green Energy Co-efficient) [35] & $\begin{array}{l}\text { Number } \\
{[0 \text { to } 1.0]}\end{array}$ \\
\hline & $\begin{array}{l}\text { ITEE (IT Equipment Energy Efficiency) } \\
\text { [43] }\end{array}$ & $\%$ (Percentage) \\
\hline & ITEU (IT Equipment Utilization) [43] & Number \\
\hline & $\begin{array}{l}\text { HVAC (Heating, Ventilation, Air- } \\
\text { conditioning) Effectiveness [42] }\end{array}$ & Dimensionless \\
\hline & Cooling System Efficiency [42] & $\mathrm{kW} / \mathrm{ton}$ \\
\hline \multirow{2}{*}{$\begin{array}{l}\text { Carbon } \\
\text { footprint }\end{array}$} & CUE(Carbon Usages Effectiveness) [35] & $\begin{array}{l}\mathrm{KgCO} 2 \text { per } \mathrm{kW}- \\
\mathrm{h}\end{array}$ \\
\hline & $\begin{array}{l}\text { DPPE (Data Center Performance Per } \\
\text { Energy) [43] }\end{array}$ & $\begin{array}{l}\text { Number } \\
{[0 \text { to } 1]}\end{array}$ \\
\hline \multirow{2}{*}{ Recycling } & e-Wastage Or IT Wastage [42] & Gm (Gram) \\
\hline & Recycling $[37,44]$ & $\%$ (Percentage) \\
\hline \multirow{5}{*}{ Productivity } & DCP (Data Center Productivity) [35] & Not Available \\
\hline & $\begin{array}{l}\text { DCeP (Data Center Energy } \\
\text { Productivity) }[24,35]\end{array}$ & Not Available \\
\hline & Analysis Tool [26] & Not Known \\
\hline & EnergyBench [26] & Numeral Rating \\
\hline & ScE (Server Compute Efficiency) [35] & $\%$ (Percentage) \\
\hline $\begin{array}{l}\text { Costing } \\
\text { Information }\end{array}$ & Energy/Power Cost [41] & $\begin{array}{l}\text { Currency } \\
\text { [according to } \\
\text { country] }\end{array}$ \\
\hline \multirow{7}{*}{ Others } & $\begin{array}{l}\text { SWaP (Space, Wattage and } \\
\text { Performance) }[24,35]\end{array}$ & Not Available \\
\hline & User Satisfaction $[11,24]$ & Number [0 to 5] \\
\hline & $\begin{array}{l}\text { Mean Opinion Score } \\
\text { (MOS) }[11,24,45]\end{array}$ & Number [1 to 5] \\
\hline & Reliability [24] & $\begin{array}{l}\text { Number } \\
{[0.0 \text { to } 1.0]}\end{array}$ \\
\hline & Air Management Metric [42] & $\mathrm{F}$ (Fahrenheit) \\
\hline & $\begin{array}{l}\text { UPS System Efficiency } \\
\text { [42] }\end{array}$ & $\%$ (Percentage) \\
\hline & $\begin{array}{l}\text { Risk Assessment } \\
{[11,24]}\end{array}$ & $\%$ (Percentage) \\
\hline
\end{tabular}




\section{FUtURE GREEN SLA (GSLA) DEFINITION}

In existing GSLAs, most of the performance indicators mainly concentrate on energy consumption issues and productivity concern in cloud and grid computing industry (Table VII). Most of the existing GSLA do not consider recycling, radio wave, toxic material usage, noise, light pollution for sustainable development. Moreover, people's interaction and IT ethics issues, such as user satisfaction, intellectual property right, user reliability, confidentiality etc are also missing in current GSLA under green computing lens. Next section discusses the proposed new performance indicators of GSLA for achieving sustainability from 3Es perspectives (Ecological, Economical and Ethical). Fig.1 shows the concepts of 3Es relationship, that ICT engineer can use as a guideline to respect all the facets of sustainable development.

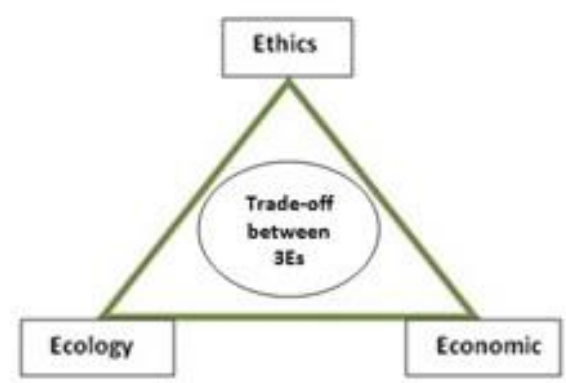

Fig. 1. 3Es for Sustainability

The proposed definition according to Fig. 1 could be "the GSLA should aggregate and satisfy all three main pillar of sustainability achievement- Ecology Pillar, Economy Pillar, and Ethics Pillar". There must be trade-offs between 3Es to achieve sustainable development under green computing domains. Under Ecology Pillar the following new indicators should take into consideration while developing new services or application in the ICT field, such as, Recycling, ICT Toxic Material Usage limit, ICT Radio Wave guideline, Pollution level and Obsolescence Indication etc. Moreover, at the same time, Economic Pillar needs to aggregate some new indicators in future GSLA;- Carbon Taxation, ICT Product Life Cycle Cost, Civil Engineering Cost, Cooling cost, Energy Cost etc. Moreover, research shows that, in most industries the green computing practice focuses on the ecological, economical point but usually neglect human's interaction and ethical aspects [37]. The use of ethics in IT and ICT field covers many new indicators such as Satisfaction level, Intellectual Property Right, Reliability, Confidentiality, Security and Privacy, Gender/Salary/Productivity Information. The ICT companies should also analyze their social responsibilities towards their customer, employee and community through developing IT Ethics program and guideline [37, 46]. All of these indicators are usually subjective and informative, thus making GSLA assessment difficult in future. On the other hand, ecological and economic indicators seem might be easy to evaluate and monitor.

In this section, this research gives some idea most of the important missing performance indicators with respect to three pillars of sustainability and this will definitely help ICT and IT service providers to develop and design their existing GSLA more greener for achieving sustainability as well as making more profit in their businesses. However, ICT engineer would face some challenges to incorporate, manage and finding the relationship between all new indicators for GSLA under three pillars of sustainability in future. To achieve sustainability, the future GSLA should aggregate and satisfy all three entities in their existing GSLA model- Ecology Pillar, Economy Pillar, Ethics Pillar. Now, it the matter of urgency that, to achieve sustainability the ICT industry need to indentify more new services from users perspective under this three pillar too. It is important to indicate that, the ICT Product Life Cycle must need to include at the first level of GSLA model as this entity have direct relationship to calculate existing ecological, economical and ethical indicators, such as carbon/GHG emission, energy consumption, recycling, energy cost, pollution level, comfort level etc [Table VII]. The ICT product life cycle and its relationships with sustainability pillars coexist while developing future GSLA. In future, ICT Product Life Cycle also needs to define as new services for achieving sustainability in the ICT industry. The whole life cycle of an ICT product consists of following four main entities, manufacturing, transportation, usage and dismantling entities. All these entities should directly connect to future GSLA design to respect global analysis of sustainable development. The total GHG emission, total energy consumption and total costing of energy could not be estimated without considering all these product life cycle entities. The interaction between ICT product life cycle and GSLA are shown in Fig.2 using UML notation [47]. 


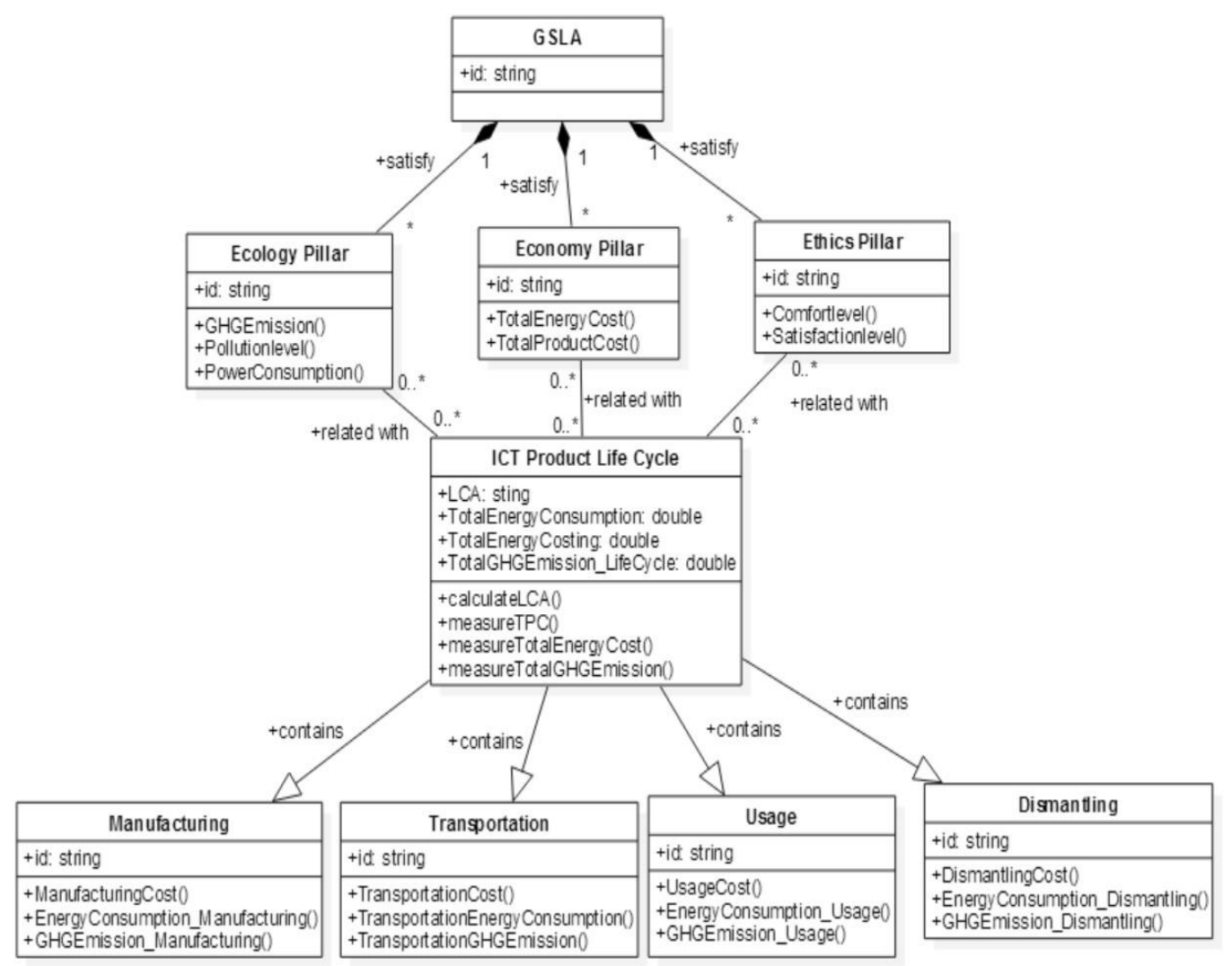

Fig. 2. Relationship between GSLA and ICT Product Life Cycle

At the bottom level of Fig.2 depicts all four main entities under ICT product life cycle. For example, manufacturing entities needs to calculate manufacturing cost, then energy/power consumption during manufacturing process and also total GHG emission during that time. The same way, the other entities would calculate their own costing, energy consumption and GHG emission. Therefore, total costing, total energy consumption and total GHG emission could be accumulated during the whole life cycle of each ICT product. Additionally, an environmental closed-loop supply (ECLS)[48] chain would need to be added with the proposed relationships as currently ICT products remanufacturing are getting importance in the industry. The ECLS chain would be helpful to improve economic and environmental performance of every product [46]. This UML notation actually shows the importance of ICT Product Life cycle assessment, while providing any new services. The next step of this research would be identifying and analyzing all new entities under $3 \mathrm{E}$ for achieving a sustainable GSLA. In future, this research would work on validation and evaluation of the proposed UML notation (Fig.2) by taking some case study on ICT product. An overall framework and survey could be designed after the case study analysis.

In addition, to define and design new green SLA (GSLA), the ICT engineer should analyses different level of cascading effect of any ICT product- direct, indirect important and indirect small effects. Moreover, finding measurement units and their assessment for all new indicators need to be defined through proper standardization and authority. At the same time, user awareness and knowledge about sustainable development should need to incorporate at different level of the society. Moreover, to reach the sustainability, industries should invest in design and planning of their products, \& also to optimize their logistic network considering the trade-off between cost and environmental effects.

\section{CONCLUSION}

This GSLA research did survey and review on different basic SLA parameters for network, compute, storage and multimedia domain of IT and ICT industry. The analysis of existing theory work on basic SLAs and GSLAs are mentioned in Table I and II. Empirical Work Review section demonstrates most of the basic SLAs performance indicators and their measurable unit for all mentioned services (Table III to Table VI). Moreover, existing GSLA survey covers most of the recent days green indicators and their measurable unit which are presented using Table VII from different computing industry. In addition, Table VII also discovers today's concerns are mainly on energy issues and productivity through the greening lens in many industries. These industries actually overlooked practicing sustainable development in their scope. This research believe, incorporating all new and existing indicators for future new GSLA might be difficult and cumbersome work for the ICT engineers. The management complexity of some proposed indicators in future GSLA would be the most challenging task. It is worth mentioning here that, ICT product life cycle need to consider at the first level of new GSLA design. The research shows the relation of ICT product life cycle with future GSLA for achieving sustainability. Some challenges exist for designing sustainable 
future GSLA such as, new performance indicators need to be defined accurately which has association with other indicators; most of the subjective, qualitative indicators related with ethics issue need standardization or governed and authorized by proper laws and directives. In addition, it is very important to mention here that the definition of GSLA is crucial in development of Green ICT solutions and requires long time to be standardized. The standardization of green indicators is one of the main issues as mentioned by ITU-T report (2012). Also, further research is necessary on monitoring the indicators which depend on human interactions. However, this research illustrates a rigorous survey and analysis to provide a new dimension and strategy for defining future GSLA under sustainability lens in ICT arena.

\section{ACKNOWLEDGMENT}

The authors would like to thank PERCCOM Consortium for giving the theme of Green SLA. The authors would like to show their gratitude and thanks to European Union for supporting PERCCOM. Few Parts of this work submitted in International SEEDS Conference 2015, Leeds Beckett University, UK.

\section{REFERENCES}

[1] L. Wu, and R. Buyya, "Service Level Agreement (SLA) in Utility Computing Systems," Performance and Dependability in Service Computing: Concepts, Techniques and Research Directions, V. Cardellini et. al. (eds), ISBN: 978-1-60-960794-4, IGI Global, Hershey, PA, USA, July 2011, pp.1-25.

[2] R. Buyya, J. Broberg, and A. Goscinsk, "Cloud Computing: Principles and Paradigm," A John Wiley \& Sons, Inc. Publication, ISBN: 978-0470-88799-8, February 2011.

[3] J. Mankoff, R. Kravets, and E. Blevis, "Some Computer Issues in Creating a Sustainable World" Computer, Vol. 41, No. 8, 2008

[4] SMART 2020 Report, "Enabling the low carbon economy in the information age," The Climate Group, GeSI, 2008.

[5] Z. S. Andreopoulou, "Green Informatics: ICT for Green and Sustainability," Journal of Agriculture Informatics (EIFTA), Vol. 3, No. $2,2012$.

[6] S. A. Baset, "Cloud SLAs : Present and Future," IBM Research, 2011, pp. 57-67.

[7] H. Lee, M. Kim, and J. W. Hong, "Mapping between QoS Parameters and Network Performance Metrics for SLA monitoring," Conference on the Asia-Pacific Network Operations and Management Symposium (APNOMS), South Korea, September 2002

[8] L. Jin, V. Machiraju, and A. Sahai, "Analysis on Service level Agreement of Web Services," Software Technology Laboratory, HP Laboratories Palo Alto, HPL-2002-180, June 2002.

[9] A. Paschke, and E. Schnappinger-Gerull, "A Categorization Scheme for SLA Metrics," Multi-Conference Information Systems (MKWI06), Passau, Germany, 2006.

[10] J. Lankinen, and J. Porras, "Survey on security profiles of existing cloud services," Wireless World Research Forum (WWRF) conference, October, 2012.

[11] Anonymous, "Green Cloud Technologies: Service Level AgreementVoice and Internet," SLA 1. 2VO, May 2013.

[12] C. Raibulet, and M. Massarelli, "Managing Non-functional aspects in SOA through SLA," $19^{\text {th }}$ International IEEE Workshop on Database and Expert Systems Application, DEXA, September 2008.

[13] V. Stantchev, and C. Schropfer, "Negotiating and Enforcing QoS \& SLAs in Grid \& Cloud Computing," $4^{\text {th }}$ International Conference on Grid and Pervasive Computing, GPC, LNCS 5529, 2009, pp. 25-35.

[14] N. J. Dingle, W. J. Knottenbelt, and L. Wang, "Service level Agreement Specification, Compliance prediction and monitoring with Performance
Trees," 22nd Annual European Simulation and Modelling Conference (ESM'08), October 2008, pp. 137-144.

[15] T. Unger, F. Leymann, S. Mauchart, and T. Scheibler, "Aggregation of Service Level Agreements in the Context of Business Processes," 12th International IEEE Enterprise Distributed Object Computing Conference, 2008.

[16] E. Marilly, O. Martinot, H. Papini, and D. Goderis, "Service Level Agreements: A main challenges for next generation network," $2^{\text {nd }}$ European Conference on Universal Multiservice Networks, ECUMN, April 2002.

[17] T. Onali, "Quality of Service technologies for Multimedia Applications in Next Generation Networks," Ph. D Thesis, University of Cagliari, Italy, 2007.

[18] H. Ludwig, A. Keller, A. Dan, and R. King, "A Service Level Agreement for Dynamic Electronic Services," IEEE international workshop on E-Commerce and Web-Based Information Systems (WECWIS), 2002.

[19] P. Hasselmeyer, H. Mersch, B. Koller, H. N. Quyen, L. Schubert, and P. Wieder, "Implementing an SLA negotiation framework," Proceedings of eChallenges Conference, 2007.

[20] White Paper, "Comparing Public Cloud: Service Level Agreements," Dimension Data, 2013.

[21] E. Wustenhoff, "Service level Agreement in the Data Centre," Sun Microsystems, April 2002.

[22] C-SIG-SLA Subgroup Members, "Cloud Service Level Agreement Standardization Guidelines," White Paper, Brussels, June 2014.

[23] S. Klingert, T. Schulze, and C. Bunse, "GreenSLAs for the energyefficient management of data centres," International Conference on Energy-Efficient Computing and Networking, May, 2011.

[24] G. von Laszewski, and L. Wang, "GreenIT Service Level Agreements," Grids and Service-Oriented Architectures for Service Level Agreements, Springer Science, LLC, 2010, pp. 78-88.

[25] Md. E. Haque, K. Le, I. Goiri, R. Bianchini, and T. D. Nguyen, "Providing Green SLAs in High Performance Computing Clouds," International Green Computing Conference, June, 2013.

[26] R. R. Harmon and N. Auseklis, "Sustainable IT Services: Assessing the Impact of Green Computing Practices," IEEE xplore, Proceeding of Portland International Centre for Management of Engineering and Technology, PICMET, August, 2009.

[27] F. H. Grupe, T. Gracia-Jay, and W. Kuechler, "Is It Time For An IT Ethics Program?," Information Management: Strategy, Systems and technologies, Aurerbach Publications, CRC Press LLC, 2002.

[28] R. Herold, Introduction to Computer Ethics, Source: http://www.infosectoday.com/Articles/Intro_Computer_Ethics.htm, Retrieved on March 2015.

[29] N. Agrawal, and K. N. Agrawal, "Current trends in Green ICT," Journal of Administration \& Governance (JOAAC), Vol. 7, No. 1, 2012.

[30] A. Amokrane, M. F.Zhani, Qi Zhang, R. Langar, R. Boutaba, and G. Pujolle, "On Satisfying Green SLAs in Distributed Clouds" $10^{\text {th }}$ International Conference on Network and Service Management (CNSM), November 2014, pp. 64-72.

[31] C. Li, A. Qouneh, and T. Li, "iSwitch: Coordinating and Optimizing Renewable Energy Powered Server Clusters," International Symposium on Computer Architecture, May 2011.

[32] K. Le, O. Bilgir, R. Bianchini, M. Martonosi, and T. D. Nguyen, "Managing the Cost, Energy Consumption, and Carbon Footprint of Internet Services," Proceedings of the ACM SIGMETRICS international conference on Measurement and modeling of computer systems, 2010, pp. 57-58.

[33] M. Nichollas, "Why Users still see Red even when SLAs are Green," Fujitsu Service Limited, United Kingdom, 2010.

[34] A. P. Bianzino, C. Chaudet, D. Rossi, and J. Rougier, "A Survey of Green Networking Research," IEEE Communication Surveys and Tutorials, Vol. 14, Issue. 1, December 2010, pp. 3-20.

[35] A. Atrey, N. Jain, and Iyengar N. Ch. S. N, "A Study on Green Cloud Computing," International Journal of Grid and Distributed Computing, Vol. 6, No. 6, 2013, pp. 93-102. 
[36] A. C. Orgerie, "A Survey on Techniques for Improving the Energy Efficiency of Large Scale Distributed Systems," ACM Computing Surveys (CSUR), Vol. 46, Issue 4, April 2014.

[37] E. Rondeau, F. Lepage, J. P. Georges, and G. Morel, "Measurements and Sustainability," Chapter 3, Green Information Technology, 1st Edition, A Sustainable Approach, Dastbaz \& Pattinson \& Akhgar, ISBN: 9780128013793, Elsevier Book, 304 pages, March 2015.

[38] SLA@SOI, Source: http://sla-at-soi.eu/, retrieved on April 2015.

[39] D. Jiang, X. Zhengzheng, and L.V Zhihan, "A multicast delivery approach with minimum energy consumption for wireless multihop networks" Journal of telecommunication Systems, 2015, pp. 1-12.

[40] D. Jiang, X. Ying, Y. Han Y, and L.V Zhihan, "Collaborative multi-hop routing in cognitive wireless networks" Journal of Wireless Personal Communications, 2015, pp. 1-23.

[41] R. L. Sawyer, "Calculating Total Power Requirement for Data Centers," White Paper, Schneider Electric white paper library, Retrieved on March 2015.

[42] P. Mathew, S. Ganguly, S. Greenberg, and D. Sartor, "Self Benchmarking Guide for Data Centers: Metrics, Benchmarks, Actions," Report of New York State Energy Research \& Development Authority (NYSERDA), July 2009.

[43] T. Shiino, "Green IT by all Parties," PhD Presentation at Nomura Research Institute, Tokyo, Japan, March 2010.

[44] N. Drouant, E. Rondeau, J. P. Georges, and F. Lepage, "Designing green network architectures using the Ten Commandments for a mature ecosystem," Computer Communications, Vol. 42, April 2014, pp. 38-46.

[45] Anonymous, "Application Note: Voice Quality Measurement, Series: Voice over IP Performance Management," Telchemy Incorporation, USA, November 2014.
[46] M. B. Uddin, M. R. Hassan, and Kazi M. Tarique, "Three Dimensional Aspects of Corporate Social Responsibility," Daffodil Intenational University Journal of Business and Economics, Bangladesh, Vol.3, No.1, January 2008.

[47] IBM Technical Libaray, "UML Basics: The Class Diagram," Source: http://www.ibm.com/developerworks/rational/library/content/RationalE dge/sep04/bell, Retrieved on April 2015.

[48] M. A. Ruimin, Y. A. O Lifei, J. I. N Maozhu, R. E. N Peiyu, and L. V Zhihan, "Robust environmental closed-loop supply chain design under uncertainty" Journal of Chaos, Solitons \& Fractals, Elesevier, November 2015.

\section{AUTHORS PROFILE}

Kohei Arai, He received BS, MS and PhD degrees in 1972, 1974 and 1982, respectively. He was with The Institute for Industrial Science and Technology of the University of Tokyo from April 1974 to December 1978 also was with National Space Development Agency of Japan from January, 1979 to March, 1990. During from 1985 to 1987, he was with Canada Centre for Remote Sensing as a Post Doctoral Fellow of National Science and Engineering Research Council of Canada. He moved to Saga University as a Professor in Department of Information Science on April 1990. He was a councilor for the Aeronautics and Space related to the Technology Committee of the Ministry of Science and Technology during from 1998 to 2000. He was a councilor of Saga University for 2002 and 2003. He also was an executive councilor for the Remote Sensing Society of Japan for 2003 to 2005. He is an Adjunct Professor of University of Arizona, USA since 1998. He also is Vice Chairman of the Commission "A" of ICSU/COSPAR since 2008. He wrote 33 books and published 510 journal papers. He is now Editor-in-Chief of IJACSA and IJISA. 